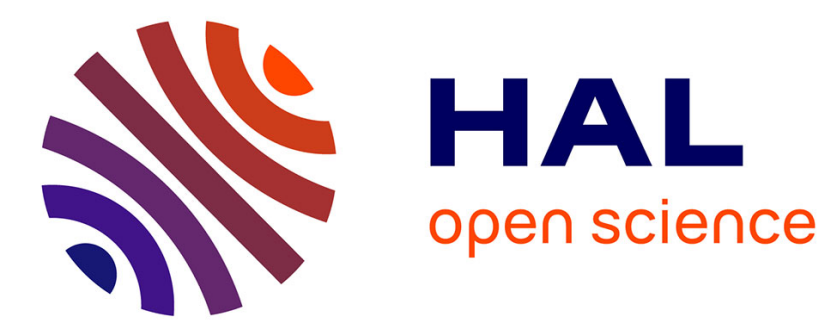

\title{
Le point sur les tétracyclines : intérêt en ophtalmologie vétérinaire
}

\author{
C. Maringue, S. Chahory
}

\section{To cite this version:}

C. Maringue, S. Chahory. Le point sur les tétracyclines : intérêt en ophtalmologie vétérinaire. Revue Vétérinaire Clinique, 2019, 54, pp.23 - 28. 10.1016/j.anicom.2018.10.003 hal-03486291

\section{HAL Id: hal-03486291 \\ https://hal.science/hal-03486291}

Submitted on 20 Dec 2021

HAL is a multi-disciplinary open access archive for the deposit and dissemination of scientific research documents, whether they are published or not. The documents may come from teaching and research institutions in France or abroad, or from public or private research centers.
L'archive ouverte pluridisciplinaire HAL, est destinée au dépôt et à la diffusion de documents scientifiques de niveau recherche, publiés ou non, émanant des établissements d'enseignement et de recherche français ou étrangers, des laboratoires publics ou privés.

\section{다)(1) $(5$}

Distributed under a Creative Commons Attribution - NonCommercial| 4.0 International 
1
LE POINT SUR LES TETRACYCLINES : INTERET EN

OPHTALMOLOGIE VETERINAIRE

AN UPDATE ON TETRACYCLINES : INTEREST IN VETERINARY OPHTHALMOLOGY

C. Maringue, S. Chahory ${ }^{1}$

\author{
${ }^{1}$ Unité d'Ophtalmologie, CHUVA, \\ Ecole Nationale Vétérinaire \\ d'Alfort, Maisons-Alfort, F-94700, France
}

Coordonnées :

0667698662

celia.maringue@vet-alfort.fr

\section{RESUME}

PROPOS

Les étracyclines sont une famille ancienne d' antibiotiques, avec un spectre large et une faible toxicié. Leur distribution tissulaire est large, dans presque tous les tissus, et de manère importante dans les tissus sécétoires. Lélimination est essentiellement énale.

Elles sont bacériostatiques par inhibition de la synthèse protique.

Elles sont utilisées dans de nombreux domaines en médecine vérinaire, et notamment en ophtalmologie.

ACTUALITES ET POINTS FORTS 
Les útracyclines posèdent de nombreuses propréés non antibiotiques : ce sont des ionophores, elles ont une action anti-inflammatoire, antiangioǵnique, inhibent l'apoptose et les enzymes proḱolytiques.

Les étracyclines topiques favorisent la cicatrisation des áosionsépittéliales chroniques spontanées, permettent de limiter l'évolution des ulçresà collaǵnases. Elles sont efficaces sur la conjonctivite chlamydienne féline et la kératoconjonctiviteà Moraxella chez les bovins.

\section{PERSPECTIVES ET PROJETS}

L'utilisation de étracyclines topiques encapsukes dans un liposome permet d'augmenter la production lacrymale sur un modele d'œil sec chez le lapin, et pourra avoir,àl'avenir, un ôle important dans la gestion de la kératoconjonctivite æ̀̀che chez le chien.

\section{MOTS CLES : TETRACYCLINES, AGENTS ANTIBACTERIENS,} OPHTALMOLOGIE

\section{SUMMARY}

\section{STATEMENTS}

Tetracyclines are old family of antibiotics, with broad spectrum and low toxicity.

They have widespread tissular distribution, in all tissues, and particularly in secretory organs. They are eliminated by renal route.

They are bacteriostatic, inhibiting bacterial protein synthesis.

They are used in many disciplines in veterinary medicine, in particular ophthalmology.

TOPICAL ISSUES 
Tetracyclines have numerous non antibiotic properties : ionophores, anti-

inflammatory, anti-angiogenic, anti-apoptotic and inhibition of

proteolytic enzymes.

Topical tetracylines promote Spontaneous Chronic Corneal Epithelial

Defects (SCCED) healing, and limit melting ulcer evolution.

They are effective against feline chlamydial conjunctivitis and bovine

Moraxella keratoconunctivitis.

PLANS AND PROSPECTS

Liposome-bound tetracycline increase lacrymal production in a rabbit dry-eye model, and will make a major contribution in canine keratoconjunctivitis sicca management.

KEYWORDS : TETRACYCLINES , ANTIBACTERIAL AGENTS, OPHTHALMOLOGY

\section{INTRODUCTION}

L'utilisation d'antibiotiques en ophtalmologie véérinaire est nécessaire dans la prise en charge de maladies oculaires mais doitêtre raisonné afin de limiter l'apparition des lésistances.

En effet, depuis avril 2016, plusieurs substances antibiotiques sontàusage restreint ou interdites d'utilisation. Une liste de substances antibiotiques d'importance critique aéf́tablie et la rélisation d'une analyse bacériologique et un antibiogramme sont obligatoires pour pouvoir les prescrire (arîédu 18/03/2016 et décret ñ2016/317 du 16/03/2016, publés dans le JORF no072 du 25/03/2016, textes n 30 et 31). 

propréés tès varées autres que l'antibiothérapie [1-3].

Ainsi, leur utilisation épandue, mais l'antibioésistance est fréquente [1]. l'oxyétracycline, la chlorétracycline, la doxycycline, et la minocycline, utilisées en médecine vérinaire ; et d'autre part la dénéclocycline, la lymécycline, la méthacycline, la roliétracycline, la glycylcycline et la tigecycline, utilisées en médecine humaine [4].

\section{PHARMACOCHIMIE}

\section{Structure}

La structure chimique des úracyclines consiste en une structure

tétracyclique correspondantàla condensation de quatre cycles (A, B, C, D)

possédant une fonction carboxamide (en C2), un groupement

L'élimination du groupement diméthylamine diminue l'activiéantibiotique, mais accrôt les propróés non-antibiotiques [1].

Les propriés biologiques peuventêtre optimisés en modifiant la zone périphérique supérieure (de C7à $\mathrm{C} 9$ du cycle $\mathrm{D}$, comme pour la minocycline et la doxycycline) [5].

105

\section{Propriétés physico-chimiques}

L'hydrosolubilié dépend des groupements polaires et de l'état d'ionisation 
doxycycline est plus liposoluble que l'oxyétracycline ou la chlorétracycline [2].

Les tétracyclines sont amphoêres, maisà pédominance basique. La doxycycline est plus acide, ce qui la rend plus difficilement utilisable en topique oculaire, en raison de l'irritation qu'elle provoque [2]. Les proprếés de chélation sont permises par diff́rents sites : les fonctions cétone (C11 et $\mathrm{C} 12)$, enol ( $\mathrm{C} 1$ et $\mathrm{C} 3)$ et carboxamide(C2) [1].

\section{Pharmacocinétique}

\section{Classification [4]:}

Les étracyclines sont claşés en trois groupes, qui different par leurs propréés pharmacocinétiques et antibiotiques.

- Le premier groupe est constituédes plus anciennes tétracyclines (útracycline, oxyétracycline, chlorétracycline, déméclocycline, lymécycline, méthacycline et roliétracycline). Elles sont plus hydrophiles que les étracyclines des autres groupes, et leur absorption est plus faible.

- Le deuxème groupe est constituée de la doxycycline et de la minocycline. Elles sont $3 a ̀ 5$ fois plus lipophiles que celles du premier groupe, leur absorption est meilleure et leur distribution plus large. De plus, leur caracère liposoluble facilite leur concentration dans les tissus glandulaires, tels que les glandes lacrymales.

- Le troisème groupe est constituéde la glycylcycline et la tigecycline, composés synthétiques avec une activié antimicrobienne in vitro contre les bacéries ésistantes aux étracyclines décrites préédemment. Ce groupe ne sera pas détailKé raison de leur non-utilisation en médecine vérinaire. 


\section{MECANISME D'ACTION - PROPRIETES ANTIBIOTIQUES [1]}

Les útracyclines diffusent passivementàtravers la paroi bacérienne, et traversent la membrane cytoplasmique par un mécanisme actif. Elles se fixent ensuite sur le site A de la sous-unié30S des ribosomes, et inhibent la transduction d'ARNm bacérien. 
+, Gram -, anárobies strictes...) ; mais de nombreuses lésistances existent

(Pseudomonas aeruginosa).

La minocycline a un spectre d'action plus large que la doxycycline.

\section{FONCTIONS BIOLOGIQUES ET PROPRIETES NON-}

\section{ANTIBIOTIQUES}

En dehors de leur activié antibiotique, les étracyclines posèdent de nombreuses propréés biologiques, et sont utilisées pour leur action antiinflammatoire, anti-proéolytique, anti-angiogénique et anti-apoptotique [5]. 
217

218
- Inhibition de l'inflammation : Les étracyclines ont un ôle antiinflammatoire majeur. Elles inhibent la migration des neutrophiles, le chimiotactisme, et l'activation des lymphocytes T [5].

- Ionophores : Les étracyclines se lient aux cations bivalents et circulent dans le plasma sous forme de complexes avec les ions $\mathrm{Ca} 2+$ ou $\mathrm{Mg} 2+$. Les étracyclines ont une importance majeure dans le fonctionnement cellulaire et biologique, notamment en tant que ionophores du calcium, qui est un second messager indispensable dans la division cellulaire, les réactions métaboliques et les processus sécétoires [2,5].

\section{- Inhibition de l'activité collagénasique : La collagénase MMP-1}

(Matrix métalloproénase de type 1) est produite par les kératocytes et les fibroblastes, et est responsable de la dégradation des collagènes de type I, II et III constituants de la cornée. Les étracyclines ont une activié anticollaǵnase, qui aéécḱcrite in vitro sur des bôlures alcalines de cornées de lapin [9].

Les mécanismes impliqués dans l'inhibition de la collagénolyse sont multiples. La doxycycline est un anti-collaǵnase efficace, par sa capacieà chélater le zinc, co-facteur néessaire au fonctionnement des MMPs. Les Étracyclines pègent notamment des réactifs dériés de l'oxygène (comme l'acide hypochlorique), ce qui diminue la quantiéde neutrophiles activés produisant des collagénases [5,10].

- Inhibition de l'angiogénèse : la minocycline et la doxycycline inhibent la néovascularisation. Uneétude a mis enévidence une diminution de l'angiogérèse tumorale par la minocycline d'un facteur de 4.5 apiès 7 jours 
jours de traitement, sur des carcinomes transplanés sur des cornées de lapin lapin [11].

223

\section{Intérêt de l'oxytétracycline dans le traitement des érosions} épithéliales chroniques spontanées (Figure 2)

En ophtalmologie canine etéquine, les ulcères cornéens sont un motif de consultation fréquent. Un ulçre superficiel (ouépithélial) se rệpithélialise en 5à répittélialise en 5 à 7 jours, gêceà un processus de cicatrisation faisant intervenir la division des cellulesépithéliales et leur migration vers la zone zone uláé [7]. 
Parfois, en raison d'une alération des mécanismes de rẹpithélialisation et

d'anomalies morphologiques de la cornée, un retard de cicatrisation est

observé. On parle d'érosionépithéliale chronique spontanée (SCCED ou

Chez le cheval, les étracyclines topiques (comme l'oxyúracycline), ont un iné̂́t par leur activié anti-proéolytique, qui, en plus de leurs propréés antibiotiques, permet la stabilisation de la matrice proúque favorisant la migration des cellulesépithéliales, et ainsi la cicatrisation desérosions q́ithéliales chroniques spontanées [14].

\section{Intérêt des tétracyclines sur les ulcères à collagénases (Figure 3)}

En médecine vérinaire, les kératites uláratives bacériennes sont fiéquentes en fréquentes en raison d'une exposition importante de la cornée aux 
traumatismes et aux pathogines.

Lorsque les micro-organismes atteignent le stroma, ils se multiplient, et peuvent produire des métalloproḱinases,àl'origine d'une ckgradation rapide de la cornée [14].

La fonction visuelle peutêtre sérèrement impacḱe, et le pronostic de conservation du globe est réservé. Un traitement médical intensif doit alors Âre rapidement mis en place.

Des étracyclines topiques et/ou sysémiques peuventêtre assocéesàun antibiotique topique de large spectre (exemple : tobramycine), afin d'inhiber la collagénolyse [15]. La doxycycline (RONAXAN ND ou DOXYVAL ND), par exemple, diminue l'activiépró́lytique in vitro de $96 \%[15]$.

Dans l'étude de Monk, la concentration dans le film lacrymal de la minocycline administtée par voie oraleà 5 poneys $(4 \mathrm{mg} / \mathrm{kg}$ deux fois par jour pendant 5 jours) n'était pas suffisante pour inhiber les MMP-2 et MMP-9 in vitro, ce qui suggère qu'une utilisation par voie topique est nécessaire pour avoir une activiéinhibitrice de la collagǵnolyse [16]. Cependant, l'utilisation topique des étracyclines seules sur les kératites (ulc̀res, abcès cornéens)àStaphylococcus pseudintermedius est déconseilke en raison d'une ésistance de plus de 94\%àcette famille d'antibiotiques [17].

Les bôlures chimiques de la cornée, notamment avec des produitsàpH basique, peuvent compromettre le pronostic visuel, par destruction des membranes cellulaires et desorganisation du collagène [7]. L'utilisation topique de étracycline sur les ulc̀res secondairesàdes bî̀lures bôlures chimiques chez le lapin accétre la cicatrisation [18]. Dans l'étude de l'étude de Szmyd, l'instillation de étracyclineà $1 \%$ deux fois par jour 


\section{Intérêt des tétracyclines dans le traitement des maladies oculaires} produisant lors de la libération des corpsét́mentaires bacériens [19]. Les chlamydies sont sensibles aux útracyclines, et, même si le traitement par voie orale (doxycycline) est fiéquemment utiliśet permet d'obtenir de bons résultats, une étracycline topique peutêtre administrée, notamment chez les chats pour qui l'administration de comprimés est difficile pour le proprétaire. Le traitement topique doitêtre poursuivi apès la ésolution des signes cliniques ; jusquâ 1à2 semaines apès,àraison de 4 applications par jour [19].

L'implication dans les conjonctivites félines de Mycoplasma spp., appartenantàla flore commensale de l'appareil respiratoire, est discuḱe ; seules des infections expérimentales sur des chatons sont rapporḱes [19]. Cependant, son đéveloppement en présence d'autres pathogènes et chez un un animal immunodépriméreste toutefois possible. Cet agent pathog̀ne est 
est sensibleà la tétracycline utilisée en topique, ouàla doxycycline par voie voie systémique [19].

335

Chez les bovins, la kérato-conjonctivite infectieuse bovine (Infectious Bovine Keratoconjunctivitis ou IBK), dueàMoraxella bovis (bacille Gram -), estàl'origine d'une conjonctivite et d'une kératite ulcérative, associéeàdes abcès cornéens. Cette affection peutévoluer vers la perforation cornéenne, et a un impactéconomique non régligeable. L'utilisation d'oxytétracycline topique peutêtre prescrite, même si l'administration par voie parent́rale, notamment sous forme longue action, est la plus courante[20].

Le même traitement peutêtre utilié pour la kératoconjonctivite àMycoplasma spp., chez les caprins (Figure 4) [20].

\section{Utilisation en devenir de nouvelles formes topiques}

La forme topique est largement utilisée en ophtalmologie ; les collyres, suspensions et pommades sont les présentations les plus courantes.

Actuellement, de nouvelles formulations sont en voie de développement, comme les liposomes.

Les liposomes sont constitués de phospholipides et contiennent en leur centre un milieu aqueux à est dépoéle principe actif. Ils améliorent les propréés pharmacocinétiques des moḱcules et permettent une diffusion lente.

L'application de étracycline topique sous forme encapsuke dans un liposome, sur un modele d'œil sec chez le lapin, permet d'augmenter les valeurs du test de Schirmer et le temps de rupture du film lacrymal, et limite les alérations histopathologiques de la cornée secondaires au déficit déficit quantitatif lacrymal [21]. Il est possible que l'utilisation de 
étracyclines sous forme modifíe puisse, dans les annéesàvenir, avoir un ôle

un ôle important dans la gestion des kérato-conjonctivites æ̀̀ches,

notamment chez le chien.

Conflit d'inết : Aucun

Remerciements : Les auteurs remercient Matthias Kohlhauer pour son aide pour la éalisation du schéma de la formule chimique des tétracyclines.

\section{BIBLIOGRAPHIE}

1. Chopra I, Roberts M. Tetracycline Antibiotics : Mode of Action, Applications, Molecular Biology, and Epidemiology of Bacterial Resistance Tetracycline Antibiotics : Mode of Action, Applications, Molecular Biology, and Epidemiology of Bacterial 
Resistance. Microbiol Mol Biol Rev [Internet]. 2001;65(2):232-260. Available from:

390 http://www.facm.ucl.ac.be/Full-texts-FACM/Vanbambeke-2006-4.pdf

391 2. Nelson ML. Chemical and Biological Dynamics of Tetracyclines. Adv Dent Res [Internet]. 1998;12(1):5-11. Available from: http://journals.sagepub.com/doi/10.1177/08959374980120011901

394 3. Gelatt KN, Gilger BC, Kern TJ. Alison Clode - Chapter 7 : Clinical Pharmacology and 395 Therapeutics - Part 2: Antibacterial Agents, Antifungal agents, and Antiviral Agents in 396 Veterinary Ophthalmology. Fifth Edit. Vol. Volume 1. 386-387 p.

4. Agwuh KN, MacGowan A. Pharmacokinetics and pharmacodynamics of the

5. Sapadin AN, Fleischmajer R. Tetracyclines: Nonantibiotic properties and their clinical implications. J Am Acad Dermatol. 2006;54(2):258-65.

401

6. Plumb DC. Veterinary Drug handbook. 7th Editio. 1313-1319 p.

7. Gelatt KN, Gilger BC, Kern TJ. Eric C. Ledbetter and Brian C. Gilger - Chapter 18 : Fifth Edit. Vol. Volume 2. 976-1049 p.

8. Hume-Smith KM, Groth AD, Rishniw M, Walter-Grimm LA, Plunkett SJ, Maggs DJ. Anaphylactic events observed within $4 \mathrm{~h}$ of ocular application of an antibiotic-containing ophthalmic preparation: 61 cats (1993-2010). J Feline Med Surg. 2011;13(10):744-51.

9. Burns FR, Stack MS, Gray RD, Parerson CA. Inhibition of Purified Collogenose from Alkali-Burned Rabbit Corneas. 1989;30(7):1569-75.

10. Ralph RA. Tetracyclines and the treatment of corneal stromal ulceration: A review. Cornea. 2000;19(3):274-7.

11. Tamargo RJ, Bok RA, Brem H. Angiogenesis inhibition by minocycline. Cancer Res. 1991 Jan;51(2):672-5.

12. Yrjanheikki J, Tikka T, Keinanen R, Goldsteins G, Chan PH, Koistinaho J. A tetracycline derivative, minocycline, reduces inflammation and protects against focal cerebral ischemia with a wide therapeutic window. Proc Natl Acad Sci [Internet]. 
1999;96(23):13496-500. Available from:

http://www.pnas.org/cgi/doi/10.1073/pnas.96.23.13496

13. Chandler HL, Gemensky-Metzler AJ, Bras ID, Robbin-Webb TE, Saville WJA, Colitz $\mathrm{CMH}$. In vivo effects of adjunctive tetracycline treatment on refractory corneal ulcers in dogs. J Am Vet Med Assoc [Internet]. 2010;237(4):378-86. Available from: http://avmajournals.avma.org/doi/abs/10.2460/javma.237.4.378

14. Gilger BC. Dennis E. Brooks, Andrew Matthews, and Alison B. Clode - Chapter 7 : Diseases of the Cornea in Equine Ophthalmology. Third Edit.

15. Ollivier FJ, Brooks DE, Kallberg ME, Komaromy AM, Lassaline ME, Andrew SE, et al. Evaluation of various compounds to inhibit activity of matrix metalloproteinases in the tear film of horses with ulcerative keratitis. Am J Vet Res. 2003 Sep;64(9):1081-7.

16. Monk CS, Jeong SY, Gibson DJ, Plummer CE. The presence of minocycline in the tear film of normal horses following oral administration and its anticollagenase activity. Vet Ophthalmol. 2018;21(1):58-65.

17. Kang MH, Chae MJ, Yoon JW, Kim SG, Lee SY, Yoo JH, et al. Antibiotic resistance and molecular characterization of ophthalmic Staphylococcus pseudintermedius isolates from dogs. J Vet Sci. 2014;15(3):409-15.

18. Szmyd L, Spector SM, Perry HD, Seedor JA, McNamara TF, Golub LM. Topical Tetracycline in the Treatment of Alkali Induced Corneal Ulceration in the Rabbit. 1986;27(3):208.

19. Gelatt KN, Gilger BC, Kern TJ. Jean Stiles - Chapter 27 : Feline Ophthalmology in Veterinary Ophthalmology. 2:1477-559.

20. Gelatt KN, Gilger BC, Kern TJ. Jacqueline W. Pearce and Cecil p. Moore - Chapter 29: Food Animal Ophthalmology in Veterinary Ophthalmology. 2:1610-74.

21. Shafaa MW, El Shazly LH, El Shazly AH, El Gohary AA, El Hossary GG. Efficacy of topically applied liposome-bound tetracycline in the treatment of dry eye model. Vet Ophthalmol. 2011;14(1):18-25. 
Légendes :

Figure 1 : Formule chimique de la tétracycline. Les sites de chélation sont repésent́s en rouge.

Figure 2 : Ulcèreàbords décolḱs chez un chien - Service d'Ophtalmologie de l'ENVA 


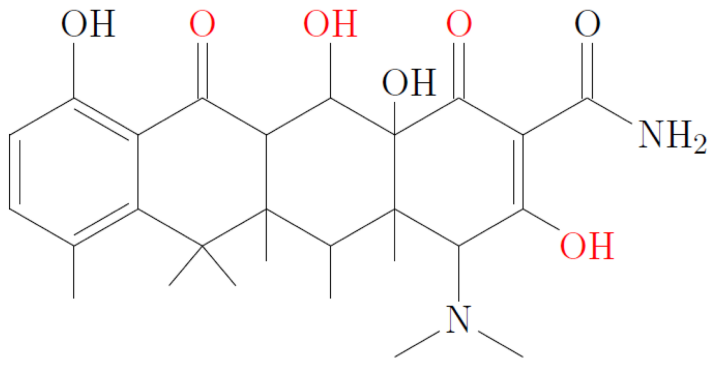




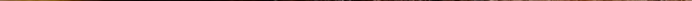




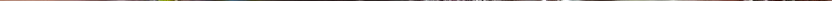


